\title{
OFICINA DE CULINÁRIA COM GRUPO DE CONTROLE AO TABAGISMO: UM RELATO DE EXPERIÊNCIA
}

\author{
Fernanda Pereira de Souza, Universidade Federal de Campina Grande (UFCG), \\ fernandapsnutri@hotmail.com \\ Gustavo Coêlho de Oliveira, Universidade Federal de Campina Grande (UFCG), \\ g_usta_1234@hotmail.com
}

Edineide Nunes da Silva, Universidade Federal de Campina Grande (UFCG), edineidens@hotmail.com

\begin{abstract}
RESUMO
O tabaco e as consequiências do seu uso são considerados importantes problemas de saúde pública, principal causa global evitável de morte, responsável por cerca de seis milhões de óbitos em todo o mundo. Nesta perspectiva, este trabalho objetiva relatar a experiência vivenciada a partir de uma oficina de culinária realizada com as mulheres participantes do grupo de combate ao tabagismo em uma Unidade de Saúde da Família. A oficina de culinária foi realizada em uma Unidade de Saúde da Família da cidade de Cajazeiras-PB junto a 06 mulheres, no período de setembro de 2016. A oficina se deu em quatro encontros, sendo o $1^{\circ}$ uma palestra, o $2^{\circ}, 3^{\circ}$ e $4^{\circ}$ a elaboração de receitas sobre bolo de fubá com goiabada, torta integral de sardinha e bolo integral de banana, respectivamente. Os resultados foram satisfatórios, visto que a evasão das participantes diminuiu significantemente durante a oficina, o que fez com que o acompanhamento com a equipe cuidadora se tornasse mais efetivo, bem como a evolução do tratamento. Conclui-se que o trabalho realizado foi de grande valia, uma vez que auxiliou de forma positiva na adesão ao tratamento para abandono do vício a partir da adoção de práticas de alimentação saudável.
\end{abstract}

PALAVRAS-CHAVE: gastronomia regional; tabagismo; oficina de culinária.

\section{CULINARY OFFICE WITH TOBACCO CONTROL GROUP: AN EXPERIENCE REPORT}

\begin{abstract}
Tobacco and the consequences of its use are considered important public health problems, the leading avoidable global cause of death, responsible for about six million deaths worldwide. In this perspective, this work aims to report the experience lived from a cooking workshop held with the women participating in the group combating smoking in a Family Health Unit. The cooking workshop was held in a Family Health Unit of the city of Cajazeiras-PB with 06 women, in the period of September 2016. The workshop took place in four meetings, the first being a lecture, the $2 \mathrm{nd}, 3 \mathrm{rd}$ and $4^{\circ}$ the elaboration of recipes on fubá cake with guava, whole sardine pie and whole banana cake, respectively. The results were satisfactory, since the
\end{abstract}


avoidance of the participants decreased significantly during the workshop, which made the follow-up with the caregiver team become more effective, as well as the evolution of the treatment. It was concluded that the work performed was of great value, since it positively helped the adherence to the treatment for abandonment of the addiction from the adoption of healthy eating practices.

KEY WORDS: Regional gastronomy; Smoking; Cooking workshop.

\title{
OFICINA DE CULINARIA CON GRUPO DE CONTROL AL TABAGISMO: UN RELATO DE EXPERIENCIA
}

\begin{abstract}
RESUMEN
El tabaco y las consecuencias de su uso se consideran importantes problemas de salud pública, principal causa global evitable de muerte, responsable de cerca de seis millones de muertes en todo el mundo. En esta perspectiva, este trabajo tiene como objetivo relatar la experiencia vivida a partir de un taller de cocina realizado con las mujeres participantes del grupo de combate al tabaquismo en una Unidad de Salud de la Familia. El taller de culinaria fue realizado en una Unidad de Salud de la Familia de la ciudad de Cajazeiras-PB junto a 06 mujeres, en el período de septiembre de 2016. El taller se dio en cuatro encuentros, siendo el $1^{\circ}$ una conferencia, el $2^{\circ}, 3^{\circ}$ y $3^{\circ} 4^{\circ}$ la elaboración de recetas sobre pastel de fubá con goiabada, torta integral de sardina y torta integral de plátano, respectivamente. Los resultados fueron satisfactorios, ya que la evasión de las participantes disminuyó significantemente durante el taller, lo que hizo que el acompañamiento con el equipo cuidador se tornara más efectivo, así como la evolución del tratamiento. Se concluye que el trabajo realizado fue de gran valor, ya que ayudó de forma positiva en la adhesión al tratamiento para abandono del vicio a partir de la adopción de prácticas de alimentación saludable.
\end{abstract}

PALABRAS CLAVE: Gastronomía regional; Tabaquismo; Taller de cocina.

\section{INTRODUÇÃO}

O tabaco continua sendo a principal causa global evitável de morte, apesar do avanço das iniciativas de combate ao seu consumo. A Organização Mundial de Saúde (OMS) estima que por ano mais de 6 milhões de pessoas morram e que ocorram prejuízos econômicos superiores a meio trilhão de dólares em decorrência do consumo de tabaco em todo o mundo (OMS, 2013). Nos próximos 50 anos, o tabagismo deve causar cerca de 450 milhões de mortes em todo o mundo. Reduzindo-se as atuais taxas de prevalência pela metade, cerca de 20 a 30 milhões de mortes prematuras poderiam ser evitadas só no primeiro quarto de século, 
e 150 milhões no segundo. Prevenindo-se que jovens iniciem o consumo de tabaco será possível reduzir o número de mortes por causas relacionadas apenas após 2050 (LANCASTER et al., 2000). Dessa forma, a única maneira de diminuir a mortalidade por causas relacionadas em médio prazo é mesmo a cessação do tabagismo, e nesta perspectiva os profissionais da saúde têm um papel fundamental (SILVA et al., 2017).

Evidências demonstram que intervenções feitas com pacientes tabagistas, ainda que curtas, menos de 3 minutos, podem fazer diferença, existindo inclusive, uma relação direta entre a intensidade da intervenção e os resultados na cessação. Mesmo aqueles que no momento da consulta não estejam inclinados a parar de fumar são, de alguma forma, influenciados (DEPARTMENT OF HEALTH AND HUMAN SERVICES, 2008).

O Instituto Nacional do Câncer (INCA) mostrou em 84 estudos realizados com fumantes em tratamento que cerca de $2 / 3$ de todas as recaídas ocorreram dentro dos primeiros 90 dias de cessação. O índice de recaída aos 6 e 12 meses, foi de $70 \%$ e chegando a 80\%, respectivamente. As características apontadas como indicativas de risco para recaída foram grau de dependência elevado, idade de iniciação ao tabagismo menor de 17 anos, convivência com fumantes, história de depressão e abuso de álcool ou outras drogas (INCA, 2013).

Apesar do sucesso das políticas de controle do tabaco no país e diminuição do consumo de fumo nos últimos 20 anos, a recaída tem sido apontada como um grande obstáculo (BRASIL, 2014).

Nesta perspectiva, o objetivo do estudo foi desenvolver uma oficina de culinária com o grupo de controle ao tabagismo, a fim de estimular a prática culinária saudável, bem como incentiva-los na adesão do tratamento, minimizando a evasão das participantes aos encontros.

\section{METODOLOGIA}

Trata-se de um estudo descritivo, caracterizado como um relato de experiência, realizado a partir de uma oficina de culinária em uma Unidade de Saúde da Família que desenvolve ações do Programa Nacional de Controle ao Tabagismo (PNCT), inserida no bairro São José, na cidade de Cajazeiras-PB, durante o estágio supervisionado do curso de graduação em Nutrição e Saúde Coletiva. O processamento das receitas foi executado na 
cozinha da própria USF. A oficina ocorreu durante o mês de setembro de 2016, com seis participantes, todas do sexo feminino e em tratamento medicamentoso nicotínico.

Comtemplou-se quatro encontros, sendo o $1^{\circ}$ uma palestra falando da relação do fumo com a alimentação e doenças associadas, o $2^{\circ}, 3^{\circ}$ e $4^{\circ}$ a elaboração de receitas, sobre bolo de fubá com goiabada, torta integral de sardinha e bolo integral de banana, respectivamente. As próprias participantes elaboravam as receitas e ao longo do preparo eram discutidas as dúvidas e esclarecidos os benefícios dos ingredientes e a importância de se introduzi-los na alimentação habitual. Durante o tempo de forno da preparação, o grupo tinha um momento com a enfermeira e a médica, relatando a experiência da semana com o tratamento e logo após, a preparação era degustada por todas.

\section{RESULTADOS E DISCUSSÃO}

A relevância do desenvolvimento de uma oficina de culinária, no que tange o incentivo de uma alimentação saudável, bem como da busca de uma melhor qualidade de vida, está ligada ao fato de se haver um mau hábito alimentar concomitante ao uso do tabaco.

No primeiro encontro a palestra contemplou a relação da alimentação e do fumo, relatando os seus malefícios, a ligação com alguns tipos de doenças, perda e ganho de peso corporal. Notou-se a inquietação das participantes nos relatos a respeito do medo de deixar de fumar diante da possibilidade de ganho de peso, um dos motivos pelo qual há resistência em abandonar o vício.

A recaída entre mulheres tem sido relatada em vários estudos, mas as razões ainda não são bem conhecidas. Embora em países ocidentais as mulheres fumem menos que os homens, elas têm mais dificuldade em parar de fumar do que eles, o que parece está relacionado à combinação de fatores biológicos, psicológicos e sociais, relata Borges e Simões-Barbosa (2008) e Mackay e Amos (2003) em seu estudo. Figueiredo (2007) relata que outro motivo observado para a recaída é a preocupação com o peso corporal. Essa preocupação pode estimular a iniciação, dificultar a cessação e aumentar a recaída ao tabagismo entre as mulheres que param de fumar.

Logo que se iniciou a atividade integrativa da oficina de culinária as ausências dos encontros quase cessaram, permanecendo praticamente com o mesmo número de 
participantes durante todos os encontros. No segundo, terceiro e quarto encontro, as participantes atuaram ativamente da produção das receitas, bastante empoderadas de conhecimento teórico e prático, o que contribuiu para que fossem elaboradas as receitas de bolo de fubá com goiabada, torta integral de sardinha e bolo integral de banana. As participantes relataram a satisfação com o conhecimento compartilhado, tornando o trabalho ainda mais gratificante.

É importante ressaltar que após o fim da oficina de culinária, observou-se a diminuição de desistências e interrupções no tratamento de algumas pacientes, confirmando deste modo, a importância da estratégia utilizada no enfrentamento do tratamento, que de certa forma motiva e fortalece o vínculo das participantes com a terapia. Considerando a complexidade que envolve o tabagismo, as dificuldades de adesão ao tratamento e o abandono do vício, observou-se que houve uma adesão significativa dos participantes quando o grupo de tabagismo estava participando da oficina de culinária.

Os estudos sugerem que a inclusão de estratégias como essas são bem vindas, uma vez que motivam os fumantes e apoiam a cessação do tabagismo, visando a melhora das taxas de adesão ao tratamento e abandono do cigarro, e consequentemente a melhoria da qualidade de vida da população (AZEVEDO et al., 2009; ECHER; BARRETO, 2008).

Deste modo, verifica-se a efetividade de uma atividade complementar junto ao tratamento de tabagistas, a oficina de culinária apresenta-se como uma estratégia positiva na tentativa de fixar o paciente no tratamento, minimizando as desistências dos encontros e as recaídas, favorecendo o sucesso do tratamento e a adesão aos hábitos alimentares saudáveis.

\section{CONCLUSÃO}

Considerando-se a prevalência do tabagismo e a dificuldade de abandono do vício, os resultados alcançados no presente trabalho confirmam a potencialidade da atividade complementar no tratamento para a cessação do uso do tabaco, uma vez que esta contribuiu positivamente para a fixação do paciente no tratamento. A oficina realizada se configura como uma opção viável e pode ser considerada como uma nova alternativa a ser executada pelas equipes multidisciplinares como estratégia de adesão ao tratamento. Conclui-se que a oficina de culinária foi uma experiência válida, que proporcionou uma troca de saberes de 
ambas as partes e que contribuiu com a adesão ao tratamento, práticas de alimentação saudável bem como a melhoria da qualidade de vida dos envolvidos.

\section{REFERÊNCIAS BIBLIOGRÁFICAS}

AZEVEDO, R. C. S.; HIGA, C. M. H.; ASSUNÇÃO, I. S. M. A.; FRAZATTO, C. R. G.; FERNANDES, R. F.; GOULART, W.; BOTEGA, N. J.; BOSCOLO,M. M.; SARTORI, R. M. Grupo terapêutico para tabagistas: resultados após seguimento de dois anos. Revista da Associação Médica Brasileira, São Paulo, v.55, n.5, p. 593-596, 2009.

BORGES, M. T. T.; SIMÕES-BARBOSA, R. H. Cigarro "companheiro": o tabagismo feminino em uma abordagem crítica de gênero. Caderno de Saúde Pública. 2008 dez.; 24(12):28342842.

BRASIL. Ministério da Saúde. Vigitel Brasil 2013: vigilância de fatores de risco e proteção para doenças crônicas por inquérito telefônico. Brasília: Ministério da Saúde, 2014.

DEPARTMENT OF HEALTH AND HUMAN SERVICES. Public Health Service.Treating Tobacco Use and Dependence: 2008 Update. Rockville: U.S. Department of Health and Human Services, 2008.

ECHER, I. C.; BARRETO, S. S. M. Determinação e apoio como fatores de sucesso para a cessação tabágica. Revista latino-americana de Enfermagem, Ribeirão Preto, v.16, n.3, p. 445-451, jun. 2008.

FIGUEIREDO, V. C. Um panorama do tabagismo em 16 capitais brasileiras e Distrito Federal: tendências e heterogeneidades [tese]. Rio de Janeiro: Universidade do Estado do Rio de Janeiro, Instituto de Medicina Social; 2007.

INSTITUTO NACIONAL DE CÂNCER (INCA). Observatório da Política Nacional de Controle do Tabaco, 2013. Disponível em:

<http://www2.inca.gov.br/wps/wcm/connect/observatorio_controle_tabaco/site/home/dados_ numeros/custos>. 
LANCASTER, T.; STEAD, L.; SILAGY, C.; SOWDEN, A. Effectiveness of interventions to help people stop smoking: findings from the Cochrane library. British Medical Journal, London, 2000; 321( 7257): 355-358.

MACKAY, J.; AMOS, A. Women and tobacco. Respirology. 2003; 8:123-130.

ORGANIZAÇÃO MUNDIAL DA SAÚDE. Relatório sobre a epidemia mundial de tabaco, 2013: aplicação de proibições de propaganda, promoção e patrocínio do tabaco. Genebra: Organização Mundial da Saúde, 2013.

SILVA, A. C.; TEIXEIRA, E. R.; GONÇALVES, S. J. C.; SOUZA, M. C. A. Tabagismo entre estudantes de profissões de saúde: prevalência, conhecimento, atitudes e opiniões. Revista de Saúde. 2017 Jan./Jun.; 08 (1): 23-27. 